\title{
Facial Image Segmentation by Integration of Level Set and Neural Network Optimization with Hybrid Filter Pre-processing Model
}

\author{
Rangayya, ${ }^{1, *}$ Virupakshappa ${ }^{2}$ and Nagabhushan Patil ${ }^{3}$
}

\begin{abstract}
Face segmentation is the process of segmenting the visible parts of the face excluding the neck, ears, hair, and beards. In this field, several methods have been developed, but none of them have been effective in providing optimal face segmentation. Hence, we proposed a novel face segmentation method known as level-set-based neural network (NN) algorithm. This method exploits a hybrid filter for the pre-processing of images, which eliminates the unwanted noises and blurring effect from the images. The hybrid filter is the combination of Median, Mean, and Gaussian filters and effectively removes the unwanted noises. Hence the images are segmented by utilizing level-set-based NN algorithm which is commonly based on the population set and effectively reduces the gap between the predicted and expected outcomes. The proposed method is compared with state-of-art methods such as Fully convolution network (FCN), Gabor filter(GF), multi-class semantic face segmentation(MSFS), and genetic algorithms (GA). From the experimental analysis, it is evident that the proposed work achieved better results comparing to other approaches.
\end{abstract}

Keywords: Neural Network; Jaccard coefficient; Dice coefficient; Segmentation; Mean; Gaussian; Hybrid filters; Level-set algorithm.

Received: 15 September 2021; Accepted: 1 December 2021.

Article type: Research article.

\section{Introduction}

Face recognition ${ }^{[1]}$ plays vital role in today's digital world. This process recognizes by extracting face features regardless of their dimensionality, orientation, or lighting conditions. Face recognition approaches generally includes knowledge-based approaches, ${ }^{[2]}$ feature invariant approaches, ${ }^{[3]}$ template matching approaches, ${ }^{[4]}$ and methods based on facial appearance..$^{[5]}$

Thus, the face segmentation and recognition have drawn considerable attention by researchers. However, can be

\footnotetext{
1 Department of Electronics \& Communication Engineering,

Sharnbasva University, Kalaburagi - 585103, Karnataka, INDIA.

2 Department of Computer Science \& Engineering, Sharnbasva

University, Kalaburagi - 585103, Karnataka, INDIA. 19110,

Jordan.

3 Department of Electrical \& Electronics Engineering, Poojya

Doddappa Appa College of Engineering, Kalaburagi - 585103,

Karnataka, INDIA.

*Email: rangu2kiran@gmail.com (Rangayya)
}

applied in smart applications like human computer interaction, querying the database, ${ }^{[6]}$ virtual reality, information security, ${ }^{[7]}$ operating system, health monitoring reports ${ }^{[8]}$ real-time online bank transactions, biometrics, etc. ${ }^{[9]}$

The efficiency of face recognition methods are highly influenced by few factors such as complex background, distortion in the expression, head lying position, the distance between the camera and the subject, lighting conditions, etc. ${ }^{[10]}$ Face recognition is an object recognition problem, ${ }^{[11-16]}$ here the face is considered as a dynamic object. Hence, it is a complex issue in computer applications.

The applications of artificial intelligence will get better results for face segmentation and recognition tasks. Consequently, image segmentation ${ }^{[17]}$ plays a vital role in face recognition using computers. Segmentation is a primary task which segments the images into pixels or various parts based on the visual understanding by the computers. There are three types of segmentations: (i) thresholding based approaches, (ii) region-based approaches, and (iii) edge-based segmentation approaches. ${ }^{[18]}$ Further, these techniques are classified as (i) structural segmentation (based on the data framework and the 
expected part of the subjected images), (ii) stochastic segmentation (depends on the pixel values of the subjected images), and (iii) hybrid segmentation (utilizes both the image frame and the discrete pixels of the image). ${ }^{[19]}$ The structural segmentation utilizes different structural components, which are physically meaningful, linearity, planarity, scattering, and uses a graph-based partitioning techniques. ${ }^{[20]}$

Face recognition plays an important role, which has been used in wide variety of modern applications. Several researchers have proposed novel techniques to improve the image recognition quality and accuracy.

\section{Related works}

Zhang et al. ${ }^{[21]}$ proposed a deep center-based dual-constrained hashing approach to restoring the discriminative face images. The discriminative and compact binary codes are generated via softmax categorization loss-oriented deep hashing. The class centers and end-to-end hashing learning are integrated simultaneously to reduce the gap between the clustering intraclass samples and the learnable class center, which creates an intra-class variance. It also enhances the Hamming distance by including a novel regularization technique among the class centers that occur in pairs. The same binary codes were generated by encouraging intra-class samples. However, an essential intra-class variation with large-scale face image recognition is a challenging task.

Face quality assessment aims at estimating the suitability of a face image for recognition. Previous work proposed supervised solutions that require artificially or human labelled quality values. However, both labeling mechanisms are errorprone as they do not rely on a clear definition of quality and may not know the best characteristics for the utilized face recognition system. Avoiding the use of inaccurate quality labels, Terhorst ${ }^{[22]}$ proposed a novel concept to measure face quality based on an arbitrary face recognition model. By determining the embedding variations generated from random subnetworks of a face model, the robustness of a sample representation and thus, its quality is estimated. This solution can be easily integrated into current face recognition systems and can be modified to other tasks beyond face recognition. However, it failed to reduce the feature dimensionality and takes a higher processing time.

The novel partition selection model was suggested by Lau et al. ${ }^{[23]}$ to recognize the modular face on occlusion issue. Due to turbulence to blur, the distortion is decomposed by achieving disentanglement. The disentanglement is regularized by implementing two ways of restoration. First, the sharp images with rich particulars are reconstructed as well as the restored image features are combined using fusion function. Second, the artifacts were suppressed; the sharp images were also reconstructed by adding perceptual and adversarial losses. However, there is a scope to improve the image detection accuracy of this method.

For machine vision applications, image recognition techniques are the foundation, but how to associate the low- level information in the image with the high-level image semantics becomes the key problem of image recognition. Predecessors have provided many model algorithms, which have laid a solid foundation for the development of artificial intelligence and image recognition. The novel work proposed by Guangxin Lou et al. ${ }^{[24]}$ improves the model to be a multilevel information fusion of the convolution calculation method, and further recovers the discarded feature information, so as to improve the recognition rate of the image. Visual geometry group (VGG) divides the network into five groups (indicating the five layers of AlexNet), yet it uses $3 * 3$ filters and combines them as a convolution sequence. This method outperformed predecessor's results, but the execution time is higher and prediction accuracy can be improved further.

$\mathrm{Li}, \mathrm{K}$ et al. ${ }^{[25]}$ suggested a novel method known as the online video object segmentation approach, which conducts the segmentation by detection structure. This approach effectively segments the video frames with high accuracy; however, the computational time is high compared to other approaches.

Kim et al. ${ }^{[26]}$ proposed a semantic-based segmentation that precisely segments and derives the facial landmark features. The classification accuracy has been improved by varying the pixel values based on the face landmark. Thus, accomplished high pixel accuracy in the face segmentation approach; however, they have used only the landmark dataset, not the real-time datasets.

Even though there are several image segmentation techniques available to date, it is a complex process to estimate the perfectly optimized segmented images to perform face recognition. Hence, we propose a novel level-set-based neural network-based face segmentation that optimally segments the faces from the dataset taken. The contributions of our work are enlisted as below.

- The images are pre-processed with a novel hybrid filter interlinked with median, mean, and Gaussian filters to eliminate unwanted noises and smoothing.

- The pre-processed images are transferred to the segmentation section and segmented by utilizing the level-set-based Neural network algorithm.

- The proposed level-set-based neural network can be exploited to acquire the optimal segmented outcomes.

The remaining part of this work is arranged as section 2 describes the proposed methodology in a wider context. Then, the experimental analysis and dataset used are discussed in section 3; section 4 discusses obtained results. Finally, we concluded our work in section 5 .

\section{Proposed active contour and level-set-based artificial neural network-based segmentation}

The proposed methodology from this article is composed of hybrid filter pre-processing and level-set-based neural network-based image segmentation, which provides better optimal face segmented outcomes, which is illustrated in Fig. 1. 


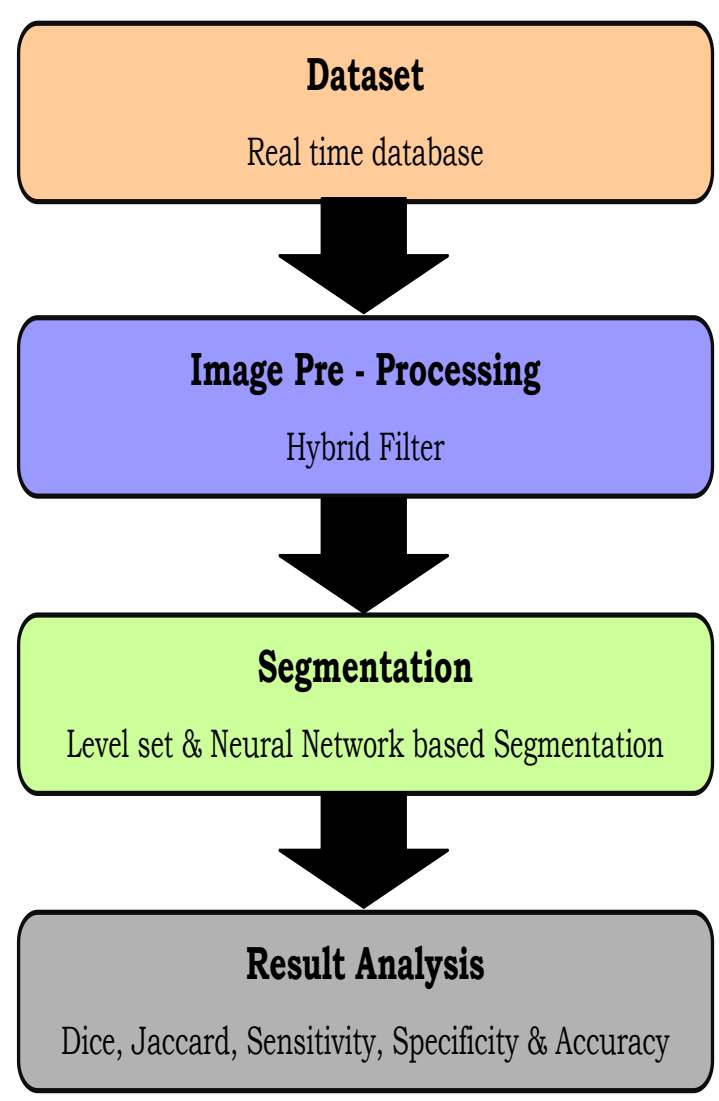

Fig. 1 Schematic diagram of the proposed methodology.

\subsection{Hybrid filter based image pre-processing}

Face recognition becomes complex due to the presence of noise and varying intensities of face images. ${ }^{[27]}$ These factors are removed with the support of filters. The proposed methodology in this article adopted hybrid filters that combine Mean, Median, and Gaussian filtering. ${ }^{[28]}$ The unwanted noises that are present in the images can be eliminated with median filtering. ${ }^{[29]}$ The median value of the pixels can be calculated by its numerical expression as follows,

$$
\operatorname{Median}(M)=\operatorname{Med}\left\{M_{i}\right\}\left\{\begin{array}{c}
M_{i}(j+1) / 2 j i s o d d \\
1 / 2\left[M_{i}(j / 2)+M_{i}(j / 2)+1\right] j i s e v e n
\end{array}\right.
$$

Here, the index of neighboring pixels is denoted as $1, M 1$, $M_{2}, M_{3}, \ldots ., M_{j}$. The median value of pixels is generated by sorting the pixels. Meanwhile, the mean filter ${ }^{[30]}$ is a sliding window-based spatial filtering technique utilized for image smoothing and noise removal. The center pixels in the windows are determined by estimating the mean value of the adjacent pixels. Numerically it can be expressed as:

$$
\operatorname{Mean}\{x, y\}=\frac{1}{M} \sum_{j=x=1}^{x+1} \sum_{x=y=1}^{y+1} a[j, l]
$$

Here, $j$ and $l$ denote the pixel locations, and $M$ denotes the used pixels from the face images. The detailed version of the noises is eliminated by a low-pass filter known as a Gaussian filter. The applied Gaussian filter ${ }^{[31]}$ can enhance the image quality and thus be used for image detection, blurring, etc. The numerical expression for the 2D Gaussian filter can be given as:

$$
\operatorname{Gau}(x, y)=\frac{1}{2 \Pi \kappa^{2}} e^{\frac{x^{2}+y^{2}}{2 \kappa^{2}}}
$$

The Gaussian filter value can be denoted as $\operatorname{Gau}(\mathrm{x}, \mathrm{y})$ the rows and columns of the matrix are indicated as $x$ and $y$. The standard deviation (SD) of the Gaussian distribution function can be given as $\kappa$. Henceforth the noise removed and the intensity adapted face images are fed as an input for the segmentation process. The proposed method utilized a novel segmentation technique known as Level-set-based neural network optimization.

\subsection{Active contour and level set based ANN-based Segmentation}

In face recognition, segmentation is used to split the digital images into many parts (group of pixels or image objects) as shown in Fig. 2.

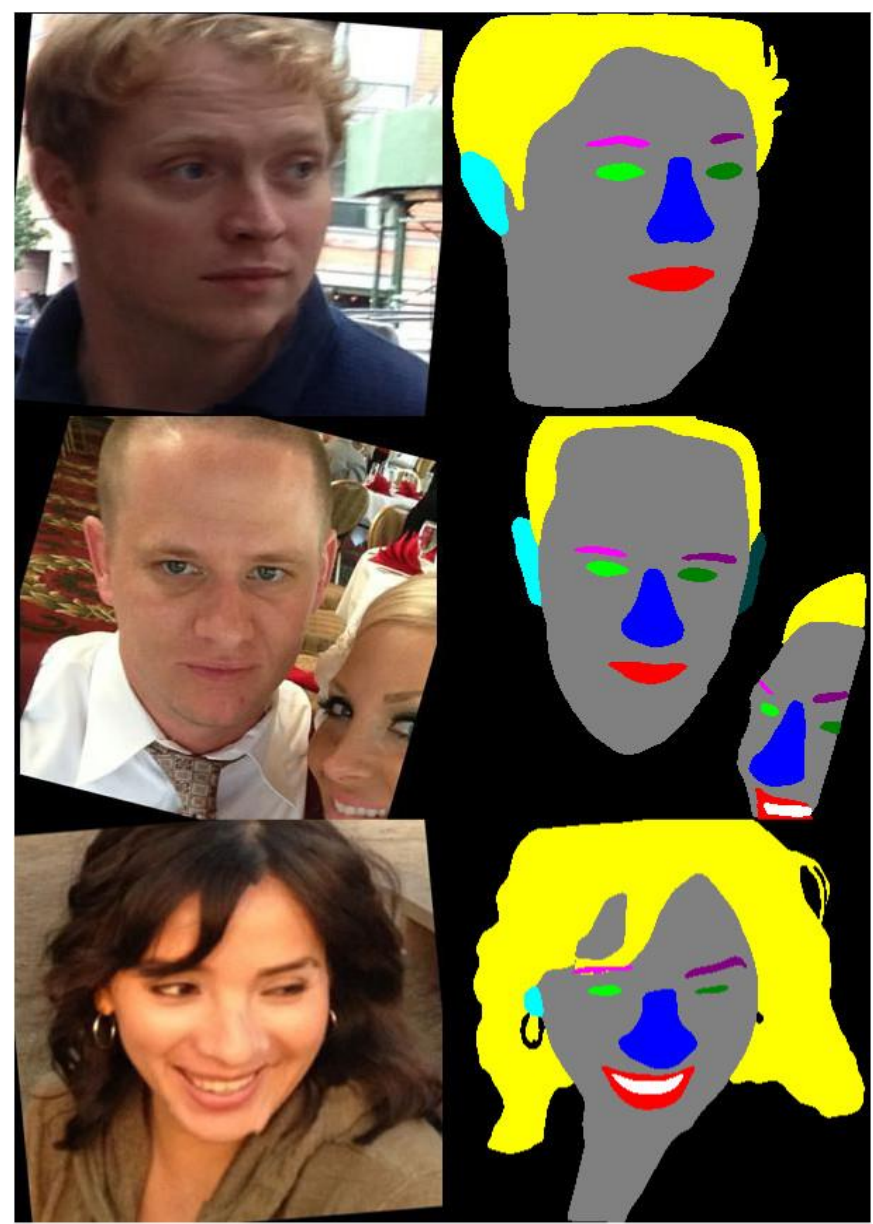

Fig. 2 Outcomes of segmentation.

\subsubsection{Active contour and Level set based segmentation}

The Active-contour method ${ }^{[37]}$ is a shape-based framework that reduces the energy function (regional properties) such as boundaries, intensities of pixels, characteristics of an object is to conduct segmentation. The contour location can be initially placed inside or outside of the region of interest arbitrarily or manually. The reduction of energy function can be used to shift the curve to the contour of the pixel while conducting the curve evolution process which is as shown in Fig. 3. 


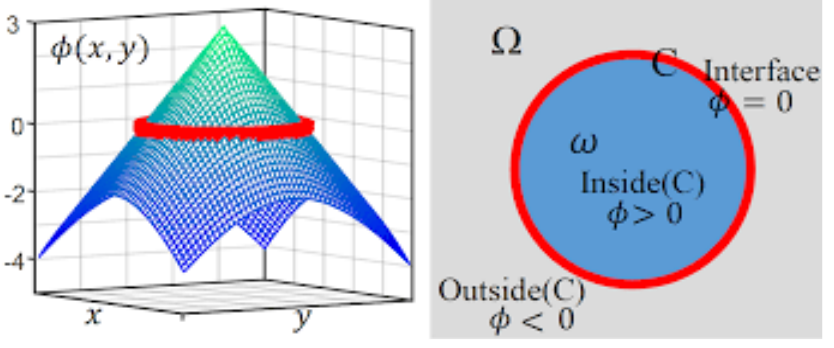

Fig. 3 The Level set for object detection.

The most commonly used curve evolution method is the marker point approach that can be used to control each point on the segmenting curve $C$ to determine the location vector $[f$, $t$ ] with the inclusion of a curve with a certain orientation $f$ and time $t$. Meanwhile, the front region can be displayed along with the marker points incorporated with either line segments (2D) or triangles (3D).

However, if the two marker points from the curve evolution come together, it might cause asymmetrical growth of the markers and lead to unlimited output with variations in curvature. Therefore, the next approach that can be applied for active shape modeling is the level-set approach. Level set approaches are a powerful mathematical tool for image segmentation.

Here, the evolving corner is completely interconnected as the zero is contour, i.e., for the unit circles, it can be defined as,

$$
\omega(\vec{x})=x^{2}+y^{2}-1, \omega(\vec{x})=0
$$

However, level-set based motion curve can be determined as,

$$
\hat{\omega}+\vec{W} \cdot \nabla \omega=0
$$

The partial temporal derivation of the inferred function $\omega$ is indicated as $\widehat{\omega}$ the velocity of the motion is determined $\vec{W}=\langle u, v, w\rangle$, and the velocity components of the $\mathrm{x}, \mathrm{y}$, and $\mathrm{z}$-axis are given as $\mathrm{u}, \mathrm{v}, \mathrm{w} . \nabla$ is defined as the spatial gradient operator. The level-set function ${ }^{[37]} \omega$ is otherwise known as the signed distance function in which it analyzes the Euclidean distance among the object and the adjacent pixel in the link. Amidst this, a positive value is assigned to the pixels that fall beyond the interface and negative for the other. Hence the level zero set is assigned to the link itself. Moreover, this level-set technique provides stable segmentation, and also the errors that occur are mostly negligible.

Since the level-set method offers framed structure, we utilize a neural network to segment the face images. A neural network is a population-based optimization method that reduces the difference between predicted and expected segmentation results.

\subsubsection{Neural network algorithm}

Neural network algorithm ${ }^{[38]}$ is a series of algorithms used to detect the inter-correlation among the data set via a process that reflects the human brain operations. Thus, the neural networks refer to the cluster of neurons that are formed either by nature or manmade. Neural networks depend on fluctuating input and provide better outcomes. A neural network consists of four phases which are listed below.

\section{$>$ Population creation}

According to the neural network algorithm, each pixel from the face image possesses weight and are denoted in vectors as shown below,

$$
\sum_{j=1}^{N} V_{i, j}^{t}=1,0<V_{i, j}^{t}<1, i=1,2, \ldots, N
$$

Here $V_{i}^{t}$ is the vector weight of each pixel and can be given as $V_{i, j}^{t}=\left[V_{i, 1}^{t}, V_{i, 2}^{t}, \ldots, V_{i, N}^{t}\right] \mathrm{N}$ denotes the total number of pixels and $t$ is the total number of epochs. The creation of population is illustrated in Fig. 4, which can be denoted as,

$$
\begin{aligned}
& A_{\text {new }, j}^{t+1}=\sum_{i=1}^{N} V_{i, j}^{t} \times A_{i}^{t}, j=1,2, \ldots \ldots, N \\
& A_{i}^{t+1}=A_{i}^{t}+A_{n e w, i}^{t+1}, i=1,2, \ldots \ldots, N
\end{aligned}
$$

The above equation $A_{i}^{t}$ is the solution of the $i^{\text {th }}$ pixel at time $=t$, and the weighted solution of the $\mathrm{j}^{\text {th }}$ pixel at time $=t+1$ is given as $A_{n e w, i}^{t+1}$.

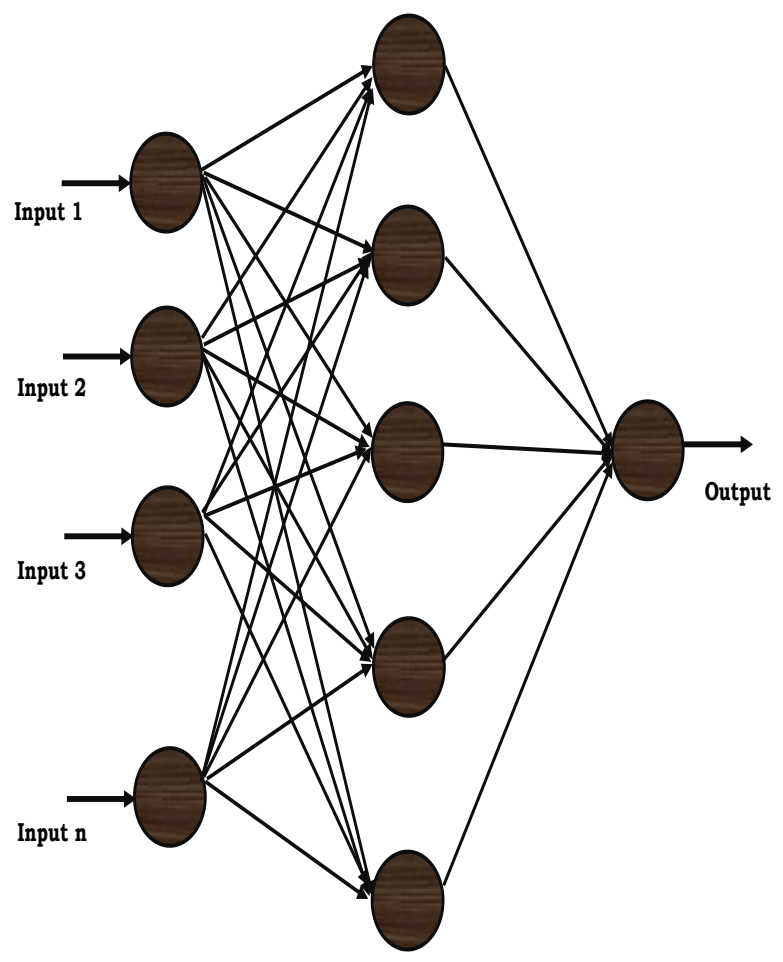

Input Layer Hidden Layer Output Layer

Fig. 4 Population creation method by neural network.

\section{$>$ Upgradation of the neural network-based weight matrix}

The weight of the pixel of the created matrix can be upgraded as,

$$
V_{i}^{t+1}=V_{i}^{t}+2 \times d \times\left(V_{o p t}^{t}-V_{i}^{t}\right), i=1,2, \ldots \ldots, N
$$

Here, $d$ is the arbitrary value that falls under 0 and 1 , and the targeted vector weight can be denoted as $V_{o p t}^{t}$. The updation takes place for the expected weight vector and the expected solution parallel. Subsequently, if the condition $A_{o p t}^{t}=$ $A_{k}^{t}(k \in[1, N\}$ for time $t$, then the weight vector $V_{o p t}^{t}=V_{k}^{t}$. 


\section{Bias operator}

The Bias operator can be updated by deeming the key parameter, which is otherwise known as modification factor $\delta$ and can be given as,

$$
\delta^{t+1}=0.99 \delta^{t}
$$

Usually, the Bias operator of NN has two portions, (i) Bias population, (ii) Bias weight matrix. To begin the Bias operation, produce an arbitrary number $N_{q}$, and that is equivalent to $\left(\delta^{t} \times D\right)$. Then a set $Q$ can be generated with an arbitrarily selecting $N_{q}$ that falls under 0 and $D$.

The lower constraints and the upper constraints are given as $\quad l c=\left(l_{1}, l_{2}, \ldots, l_{N}\right) \quad$ and $\quad u c=\left(u_{1}, u_{2}, \ldots, u_{N}\right)$ correspondingly.

Hence the population bias can be determined as,

$$
A_{i, Q(s)}^{t}=l_{Q(s)}+\left(u c_{(s)}-l c_{(s)}\right) \times e, s=1,2, \ldots, N_{Q}
$$

Here, $e$ is an arbitrary number that falls under 0 and 1 . The $N_{v}$ is an arbitrary number that can be generated for the weight matrix of the pixel and is equivalent to $\left(\delta^{t} \times N\right) . N_{v}$ lies between 0 to $N$ by choosing arbitrarily from a set $R$. Hence the bias weight matrix can be expressed as

$$
V_{i, Q(r)}=g, r=1,2, \ldots, N_{v}
$$

Here $g$ represents the arbitrary number and falls under 0 to 1 .

\section{Operation of transfer function}

The transfer function is used in NN to transmit the presently available solution to an updated location to create an optimal solution along with the surviving optimal solution as shown below,

$A_{i}^{t+1}=A_{i}^{t+1}+2 \times b \times\left(A_{o p t}^{t}-A_{i}^{t+1}\right), i=1,2, \ldots, N$

Here $\mathrm{b}$ shows an arbitrary number and lies in the range of 0 to 1 .

The Pseudo-code for Neural Network Algorithm is given below.

\begin{tabular}{l}
\hline Pseudo-code for Neural Network Algorithm \\
Initialize the pixel population and weight matrix, which \\
includes $N$ solutions \\
Estimate the fitness function for each solution and \\
estimate the optimal solution and weight \\
Repeat \\
Create the new pixel population of $A^{t+1}$ \\
Update the weight matrix of pixel $V^{t+1}$ \\
For each pixeli $\in N$, do \\
If arb $\leq \delta^{t}$ \\
Conduct the bias operator to upgrade the solution \\
$A_{i}^{t+1}$ and pixel weight matrix $V_{i}^{t+1}$. \\
Else \\
Conduct the transfer function operator to upgrade the \\
solution $A_{i}^{t+1}$ \\
End if \\
End for \\
Create the medication parameter $\delta^{t+1}$ \\
Estimate the fitness value for each pixel solution and \\
Evaluate the optimal solution $A_{o p t}^{t+1}$ and optimal weight \\
of $A_{o p t}^{t+1}$
\end{tabular}

Until satisfies the stopping criteria

Obtain the face segmented output

End

Finally, our proposed method accomplished highly accurate face segmentation by combining the level-set and neural network algorithms. Fig. 4 illustrates the pseudo-code used for the NN. In addition, the proposed method attains a precise, stable, and reduced gap between the predicted and expected face segmented images and structured frames by incorporating the Active contour level set and ANN. The process involved in the proposed segmentation stage is illustrated in Fig. 5.

\section{Experimental analysis}

This section is used to explain the performance analyses that are conducted to justify our proposed approaches. Then the results are compared with some of the latest works of face segmentation. It also includes the description of the dataset used and performance metrics.

\subsection{Dataset description and preprocessing using CLAHE}

The experimental analysis of our proposed work is carried on the MIT-CBCL and FEI datasets, including 85 frontal face images. ${ }^{[39]}$ Moreover, the faces are not organized in the same alignment and also contains faces of various ethnicity, gender, and ages. Some of the sample images of taken datasets are shown in Fig. 6.

The input images collected from the database are given to pre-processing unit where pre-processing is carried out using a hybrid technique; the input image and output images of the pre-processing unit are shown in below Fig. 7.

\subsection{Segmented output}

The proposed method attains a precise, stable, and reduced gap between the predicted and expected face segmented images and structured frames by incorporating the Active contour level set and ANN. The process involved in the proposed segmentation stage is illustrated in Fig. 5. The segmented output of our proposed work is shown in Fig. 8. From the figure, it is indisputably known that the proposed work precisely segmented the face images with higher accuracy, sensitivity, specificity, dice coefficient, and jaccard coefficient.

\section{Result and Discussion}

The performance analysis of our proposed work is conducted with some of the performance metrics such as sensitivity, specificity, accuracy, Dice coefficient, ${ }^{[40,41]}$ Jaccard coefficient $^{[42,43]}$ and compared with some of the prior works such as FCN, ${ }^{[44]} \mathrm{GF},{ }^{[45]} \mathrm{MSFS},{ }^{[46]} \mathrm{GA} .{ }^{[47]}$ The following section of this article encloses the description of performance metrics and their comparative analyses.

\subsection{Performance metrics description}

The performance metrics used for our work and its 


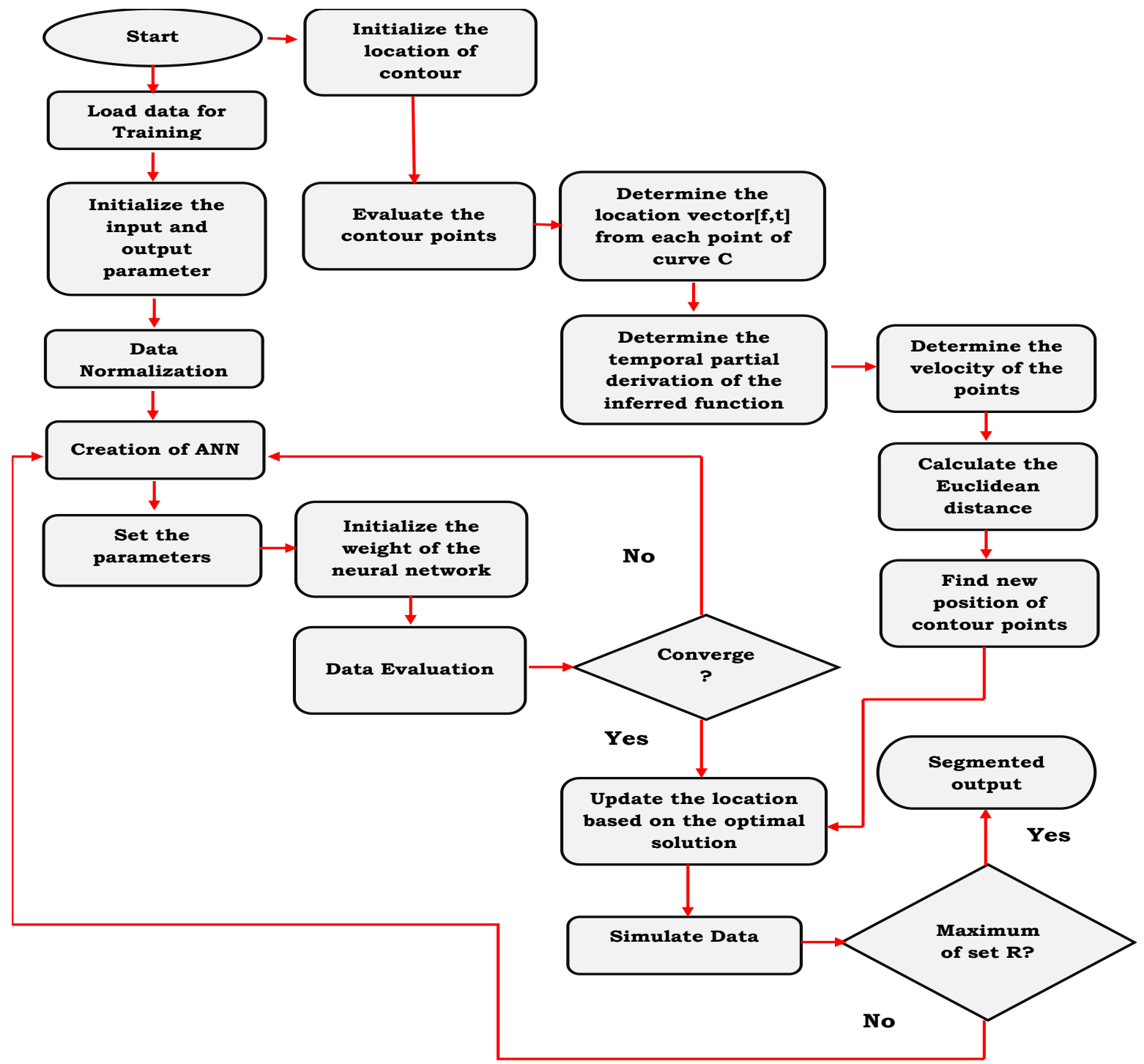

Fig. 5 Segmentation process of our proposed Active contour and Level set based ANN approach.

descriptions are listed in the following section.

\section{Sensitivity}

It is determined as the rate of pixels that are exactly segmented from the face images.

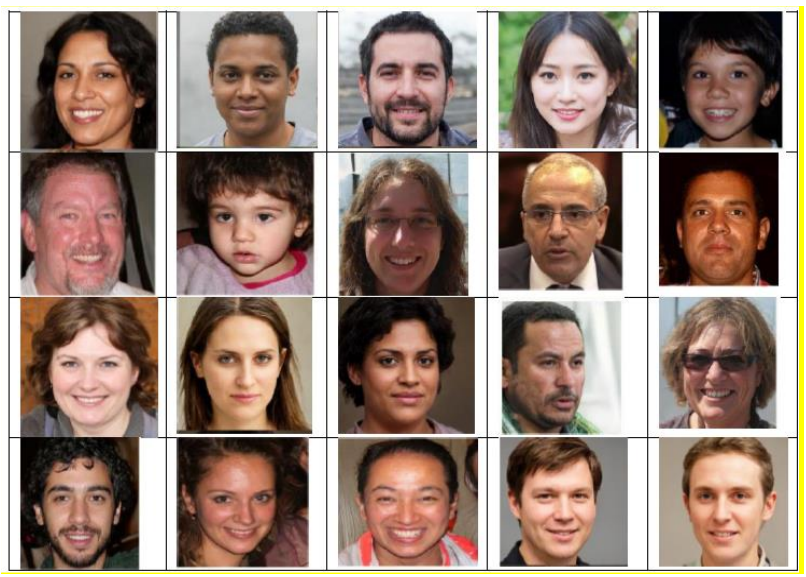

Fig. 6 Sample images from the dataset.

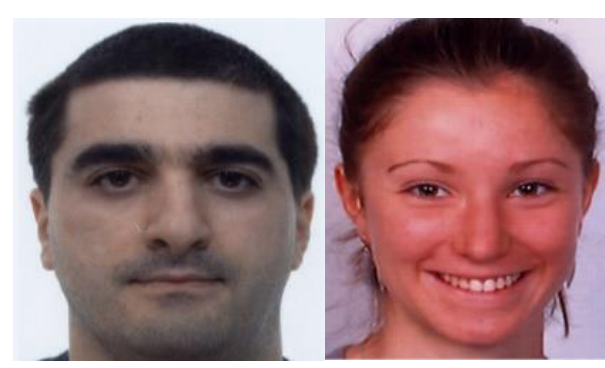

a) Sampled images from the database.

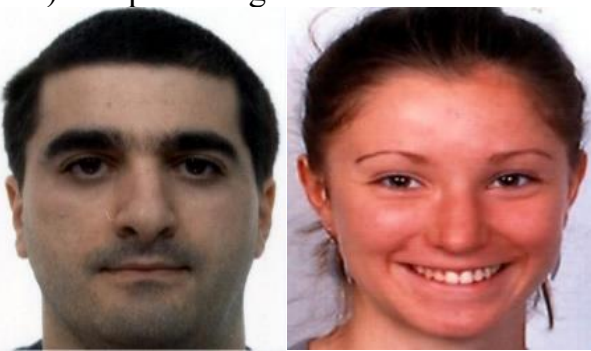

b) output image after pre-processing using Hybrid Filter technique.

Fig. 7 A hybrid filtering technique carries pre-processing. 


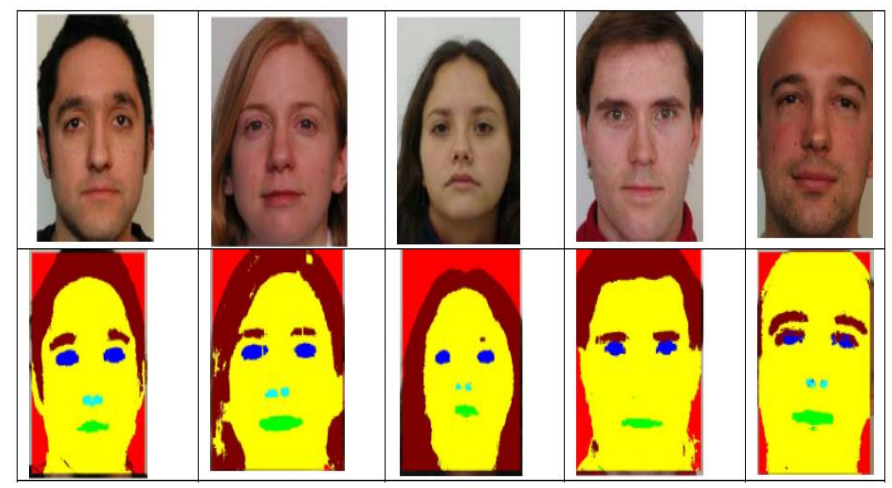

Fig. 8 The segmented output of our proposed work.

$$
\text { sensitivity }=\frac{\text { Truepositive }}{\text { Truepositive }+ \text { Falsenegtives }}
$$

\section{Specificity}

It is defined as the rate at which the normal pixels from the face images are exactly segmented.

$$
\text { Specificity }=\frac{\text { Truenegative }}{\text { Truenegative }+ \text { Falsepositive }}
$$

\section{Accuracy}

The accuracy of face image segmentation can be determined as the rate of correctly segmented faces from the total number of datasets examined.

Accuracy $=$

$$
\text { (Truepositive+TrueNegative) }
$$

$\overline{\text { Truepositive }+ \text { Truenegative }+ \text { False } \vec{f} \text { positive }+ \text { Falsenegative }}$

\section{Dice coefficient}

Dice coefficient is a statistical tool that measures the similarity among the original and detected face images. This is the important index for the validation of image segmentation that utilizes the artificial intelligence technique. it is otherwise known as the Dice similarity coefficient or Sorensen-Dice index. ${ }^{[48,49]}$

\section{Jaccard Coefficient}

The Jaccard Coefficient usually compares the original face image and the segmented face images to observe their similarity. The higher the Jaccard coefficient percentage, the more similar the two images are.

$$
\begin{gathered}
\text { Jaccard Coefficient }= \\
\frac{\text { thenumberofpixelsinbothimages }}{\text { thenumberofpixelineitherset }}
\end{gathered} \times 100
$$

\subsection{Comparative analysis}

Table 1 illustrates the comparative analysis of our proposed work with FCN, ${ }^{[44]} \mathrm{GF}^{\left[{ }^{[45]}\right.} \mathrm{MSFS}^{\left[{ }^{[46]}\right.} \mathrm{GA}^{[47]}$ in terms of accuracy, sensitivity, and specificity.

The bar chart has been plotted from these obtained values, as shown in Figs. 9, 10 and 11.

From Fig. 9, it is observed that the prior work FCN shows $88 \%$, GF exhibits $92 \%$, MSFS possesses $90 \%$, and the GA shows $94 \%$ of accuracy. On the other hand, our proposed work significantly shows the rise inaccuracy of about $98 \%$, higher than all the other approaches. Thus, the approach in this article outperforms all the work in terms of accuracy by segmenting the face images.

Table 1. Comparison of proposed work with an existing method of Accuracy, Sensitivity, Specificity.

\begin{tabular}{cccc}
\hline Methods & Accuracy $(\%)$ & Sensitivity $(\%)$ & Specificity $(\%)$ \\
\hline FCN & 88 & 90 & 88 \\
GF & 92 & 91 & 90 \\
MSFS & 90 & 88 & 78 \\
GA & 94 & 93 & 92 \\
PROPOSED & 98 & 96 & 94 \\
\hline
\end{tabular}

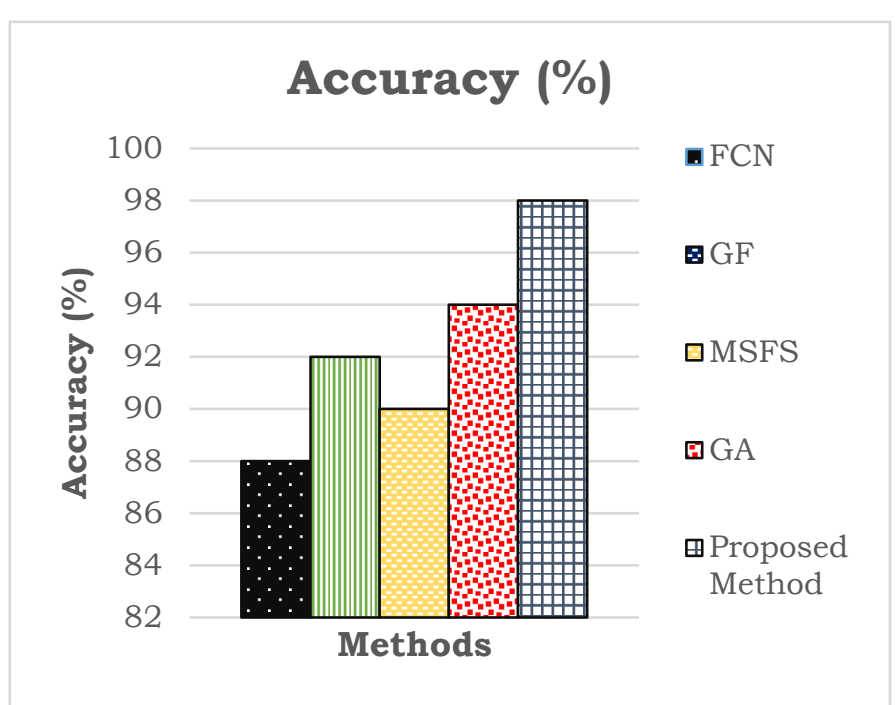

Fig. 9 Comparative analysis in terms of accuracy.

Fig. 10 represents the comparative analysis of our proposed with other existing approaches in terms of sensitivity. The sensitivity values of state-of-art works, FCN, GF, MSFS, and GA are $90 \%, 91 \%, 88 \%$, and 93\%, respectively. However, our proposed work shows $96 \%$ of sensitivity which shows the precise rate of the highly segmented face from the datasets.

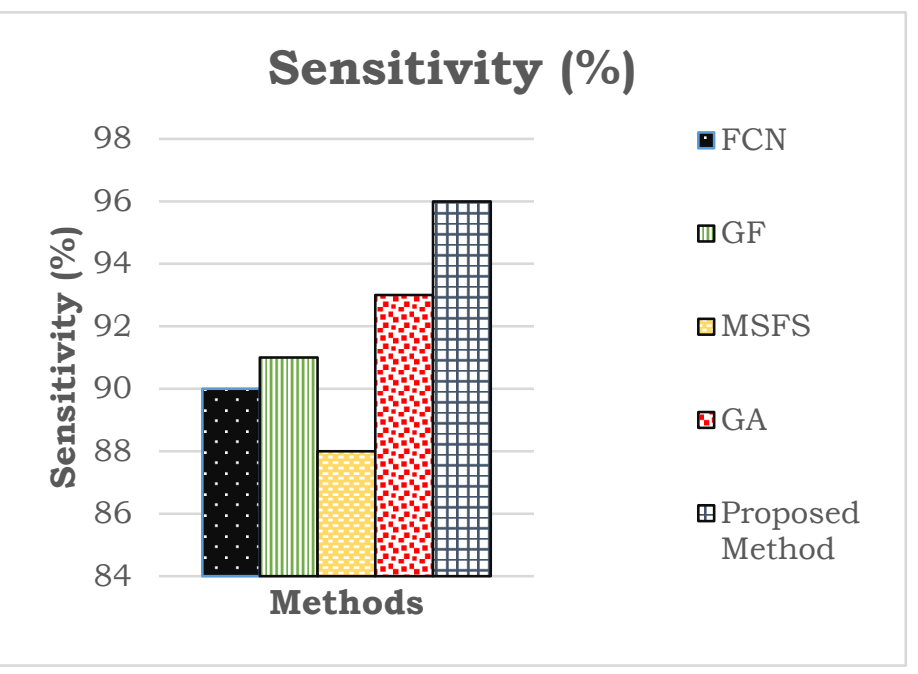

Fig. 10 Comparative analysis in terms of sensitivity. 
Fig. 11 depicts the comparative analysis of our proposed work with state-of-art approaches in terms of specificity. The specificity values of FCN, GF, MSFS, and GA are observed as $88 \%, 90 \%, 78 \%$, and $92 \%$, respectively. In the meanwhile, our proposed approach provides $94 \%$ of specificity. That shows that the normal pixels are segmented precisely from the subjected face images.

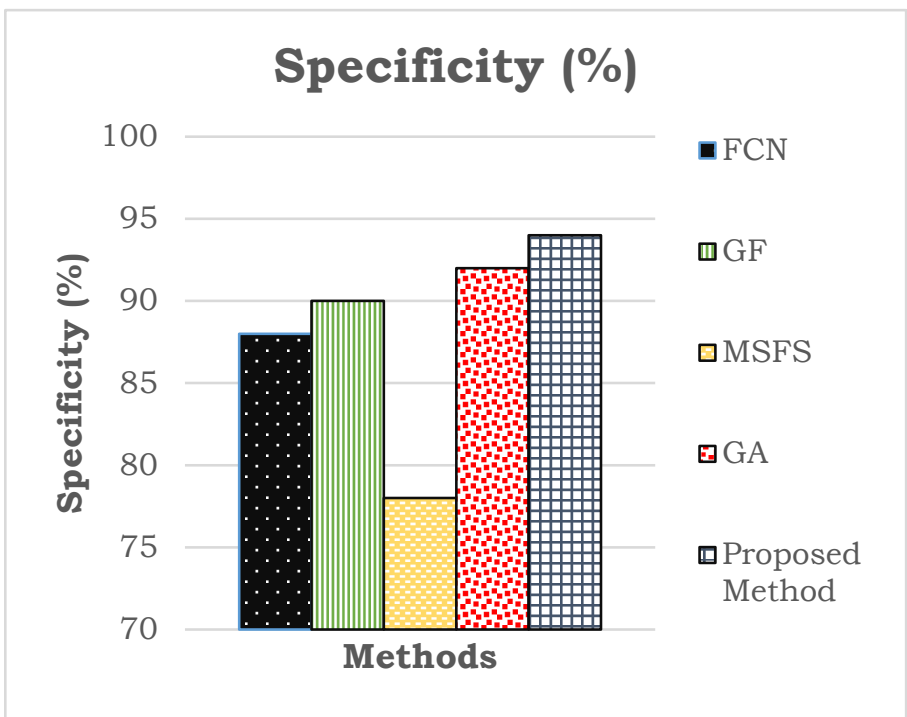

Fig. 11 Comparative analysis in terms of specificity.

The overlapping parameters such as Jaccard and Dice coefficients are evaluated against the proposed and state-ofthe-art methods. The obtained results are tabulated in Table 2 . The bar charts of the same are shown in Figs. 12 and 13.

Table 2. Comparison of proposed work with the existing method in terms of Jaccard Coefficient, Dice Co-efficient.

\begin{tabular}{ccc}
\hline Methods & $\begin{array}{c}\text { Dice } \\
\text { Coefficient(\%) }\end{array}$ & $\begin{array}{c}\text { Jaccard } \\
\text { Coefficient }(\%)\end{array}$ \\
\hline FCN & 80 & 65 \\
GF & 71 & 48 \\
MSFS & 74 & 58 \\
GA & 83 & 63 \\
PROPOSED & 89 & 71 \\
\hline
\end{tabular}

Fig. 12 shows the comparative analysis of our proposed work with the other state-of-art approaches. The figure shows that the proposed work exhibits a higher Dice coefficient of about $71 \%$, and other approaches FCN, GF, MSFS, and GA exhibit $65 \%, 48 \%, 58 \%$, and $63 \%$ correspondingly. Thus, proposed work statistically measures the similarity between the original and segmented images precisely.

Fig. 13 illustrates the comparative analysis of our proposed work in terms of the Jaccard coefficient. The figure shows that the proposed work possesses $89 \%$ of the Jaccard coefficient, and the state-of-artworks FCN, GF, MSFS, and GA possess $80 \%, 71 \%, 74 \%$, and $83 \%$ of the Jaccard coefficient

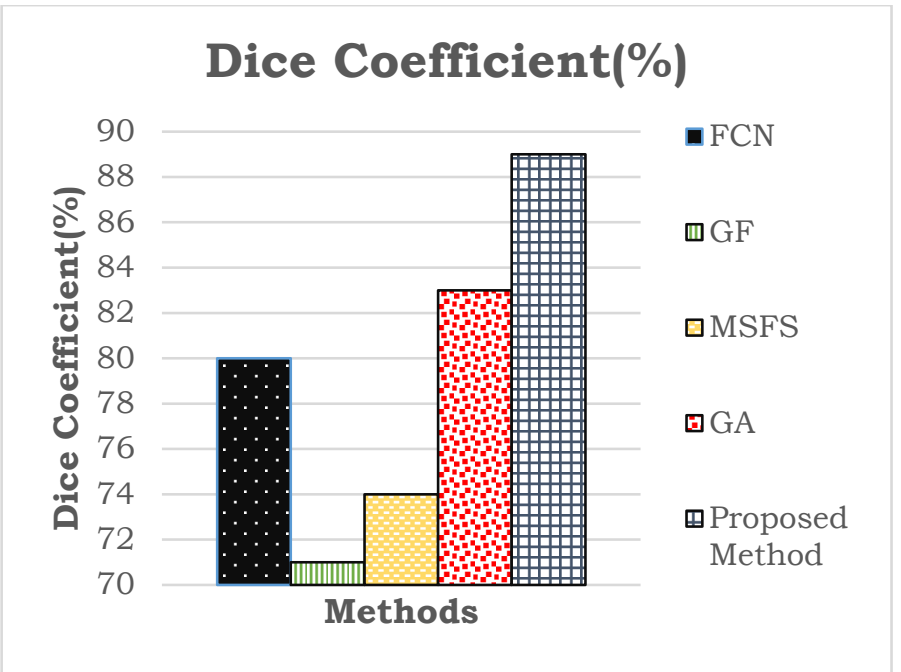

Fig. 12 Comparative analysis in terms of Dice coefficient.

correspondingly. The higher percentage of our proposed work shows that the segmented output of our proposed work is more or less similar to the original images.

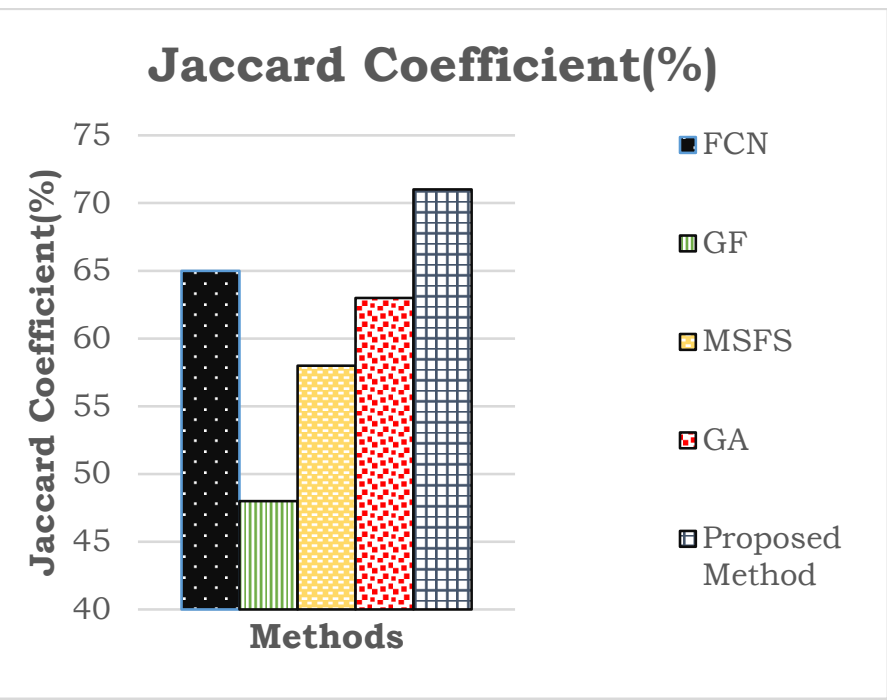

Fig. 13 Comparative analysis in terms of Jaccard coefficient.

\section{Conclusion}

This article presented a novel face segmented approach known as an active contour and level-set-based neural network algorithm that effectively segmented the subjected images from the datasets. The images were subjected to preprocessing, which exploited hybrid filters to smoothen the face images. It also removes unwanted noises. The hybrid filters are significantly composed of Mean, Median, and Gaussian filters, which effectively remove the unwanted noises. Finally, the smoothened images were fed into the image segmentation section. The proposed method effectively segments the images by utilizing the active contour and level-set-based neural network algorithm. The experimental analysis was compared with the other prior approaches such as FCN, GF, MSFS, and GA. The comparative study shows that our proposed approach has high accuracy, sensitivity, specificity, dice coefficient, and 
Jaccard coefficient of about $98 \%, 96 \%, 94 \%, 71 \%$, and $89 \%$, respectively.

\section{Acknowledgments}

We would like to thank our former guide Late Dr. Basavaraj Amarapur, for the constant guidance and support.

\section{Conflict of interest}

There are no conflicts to declare.

\section{Supporting information}

Not available.

\section{References}

[1] M Jain, K Anil., and Stan Z. Li, Handbook of face recognition., 2011, 1, New York: Springer.

[2] Liming Zhang and P. Lenders, Fourth International Conference on Knowledge-Based Intelligent Engineering Systems and Allied Technologies. Proceedings (Cat. No.00TH8516), 2000, 1, 117-120, doi 10.1109/KES.2000.885772.

[3] Z. Li, U. Park and A. K. Jain, IEEE T. Inf. Foren. Sec., 2011, 6, 1028-1037, doi: 10.1109/TIFS.2011.2156787.

[4] J. Wang and H. Yang, Congr. Image Signal Process., 2008, 575-579, doi: 10.1109/CISP.2008.270.

[5] T. Ahonen, M. Pietikainen, A. Hadid and T. Maenpaa, Proceedings of the 17th International Conference on Pattern Recognition, 2004, 3, 153-156, doi: 10.1109/ICPR.2004.1334491 [6] S. K. Veerashetty, International Journal of Innovations in Scientific and Engineering Research (IJISER), 2021, 8, 141-148. [7] A. R. L. Biradar, and V. Burkpalli, Int. J. Comput. Appl., 2019, 1-10, doi: 10.1080/1206212X.2019.1692442.

[8] Virupakshappa, Basavaraj, Health Technol., 2019, 9, 701-713, doi: 10.1007/s12553-018-00288-y.

[9] L. Torres, In Proc. of the 5th International Workshop on Image Analysis for Multimedia Interactive Services, WIAMIS, 2004, 21, 23.

[10] Lin, Shang-Hung, Informing Sci. Int. J. Emerg. Transdiscipl., 2000, 3, 1 -7.

[11] D. Barik, and M. Mondal, 2nd International conference on education technology and computer, IEEE, 2010, 2, V2-170.

[12] O. M. Parkhi, A. Vedaldi, and A. Zisserman, published in BMVC, 2015, doi: 10.5244/c.29.41

[13] A. B. Virupakshappa, A. Nandi, N. Sujatha, R. Menaka, Alex J. (eds) Computational Signal Processing and Analysis. Lecture Notes in Electrical Engineering, 2018, 490, doi: 10.1007/978981-10-8354-9 21.

[14] K. S. Priyanka, and G. Ravikumar, International Journal of Innovations in Scientific and Engineering Research (IJISER), 2015, 2, 57-72.

[15] A. R. L. Biradar, Health Technol., 2020, 10, 231-247, doi: 10.1007/s12553-018-00289-x.

[16] Sachin Kumar Veerashetty, International Journal of Innovations in Scientific and Engineering Research (IJISER), 2021, 8, 141-148.
[17] S. A. Sirohey, Human face segmentation and identification, 1998.

[18] M. R. Khokher, A. Ghafoor, and A. M. Siddiqui, IET Image Process., 2013, 7, 201-211.doi: 10.1049/iet-ipr.2012.0082.

[19] S. G. Kaur, and R. Kaur, Int. J. Comput. Sci. Eng., 2013, 5,109-112.

[20] Rao, Preeti, Thallam Prasad Vinutha, and Mattur Ananthanarayana Rohit, Transactions of the International Society for Music Information Retrieval, 2020, 3, doi: 10.5334/tismir.64. [21] M. Zhang, X. Zhe, S. Chen, and H. Yan, Pattern Recogn., 2021, 107976, doi: 10.1016/j.patcog.2021.107976.

[22] P. Terhorst, J. N. Kolf, N. Damer, F. Kirchbuchner, and A. Kuijper, In Proceedings of the IEEE/CVF Conference on Computer Vision and Pattern Recognition, 2020, 5651-5660, doi:10.1109/CVPR42600.2020.00569.

[23] C. P. Lau, C. D. Castillo, and R. Chellappa, IEEE Trans. Biom. Behav. Identity Sci., 2021, 3, 240-251, doi: 10.1109/TBIOM.2021.3058316.

[24] G. Lou, and H. Shi, China Commun., 2020, 17, 117-124.

[25] K. Li, W. Tao, and L. Liu, IEEE Access, 2019, 7, $107602-$ 107615, doi: 10.1109/ACCESS.2019.2933479.

[26] H. Kim, H. Kim, J. Rew, and E. Hwang, IEEE Access, 2010, 8, 116163-116175, doi:10.1109/ACCESS.2020.3004359.

[27] P. Majumdar, S. Mittal, R. Singh, M. Vatsa, Proceedings of the IEEE/CVF International Conference on Computer Vision (ICCV) Workshops, 2021, 3786-3795.

[28] A. Bekir, and O. K. M. Salman, Sakarya University Journal of Computer and Information Sciences, 2020, 3, 334-342, , doi: 10.35377/saucis.03.03.785749.

[29] P. Li, X. Liu, and H. Xiao, Quantum Inf. Process., 2018, 17, 1-25, , doi: 10.1007/s11128-018-1826-9.

[30] B. B. Ahamed, D. Yuvaraj, and S. S. Priya. In 2019 International Conference on Computational Intelligence and Knowledge Economy (ICCIKE), 2019, 806-810.

[31] I. Agustina, F. Nasir, and A. Setiawan, Int. J. Comput. Appl., 2017, 177, 15-19, doi: 10.5120/ijca2017915755.

[32] M. Mueller, K. Segl, and H. Kaufmann, Pattern recogn., 2004, 37, 1619-1628, , doi: 10.1016/j.patcog.2004.03.001.

[33] G. Iannizzotto, and L. Vita, IEEE Trans. Image Process. 2000, 9, 1232-1237, doi; 10.1109/83.847835.

[34] A. Xu, L. Wang, S. Feng, and Y. Qu, In 2010 Third International Conference on Intelligent Networks and Intelligent Systems, 2010, 703-706, , doi: 10.1109/ICINIS.2010.181.

[35] W. Lin, X. Li, Z. Yang, L. Lin, S. Xiong, Z. Wang, X. Wang, and Q. Xiao, Fractals, 2018, 26, 1840003, doi: 10.1142/S0218348X18400030.

[36] D. Gupta, and R. S. Anand, Biomed Signal Process. Control 2017, 31, 116-126, doi: 10.1016/j.bspc.2016.06.012.

[37] P. Ghosh, M. Mitchell, and J. Gold, Evol. Intell., 2010, 3, 111, doi: 10.1007/s12065-010-0036-X.

[38] Y. Zhang, Z. Jin, and Y. Chen, Knowl-Based Syst., 2020, 187, 104836, doi: 10.1016/j.knosys.2019.07.007.

[39] K. Khan, M. Mauro, R. Leonardi, Proc. Int. Conf. Image Process., 2015, 10.1109/ICIP.2015.7350915.

[40] B. A. Skourt, A. E. Hassani, and A. Majda, Procedia Comput. 
Sci., 2018, 127, 109-113, doi: 10.1016/j.procs.2018.01.104.

[41] R. R. Shamir, Y. Duchin, J. Kim, G. Sapiro, and N. Harel, arXiv preprint, 2019, arXiv: 1906.11031.

[42] J. Bertels, T. Eelbode, M. Berman, D. Vandermeulen, F. Maes, R. Bisschops, and M. B. Blaschko, In International Conference on Medical Image Computing and ComputerAssisted Intervention, 2019, 92-100.

[43] V. Yeghiazaryan, and I. Voiculescu, Department of Computer Science, University of oxford, 2015.

[44] Y. Nirkin, I. Masi, A. T. Tuan, T. Hassner, and G. Medioni, 13th IEEE International Conference on Automatic Face \& Gesture Recognition, 2018, 98-105, doi: 10.1109/FG.2018.00024. [45] H. A. Li, J. Fan, J. Zhang, Z. Li, D. He, M. Si, and Y. Zhang, Math. Probl. Eng., 2021, doi: 10.1155/2021/6620742.

[46] K. Khan, M. Attique, I. Syed, and A. Gul., Symmetry, 2019, 11, 770, doi: 10.3ssss390/sym 11060770 .

[47] V. Jaiswal, V. Sharma, and S. Varma., Telkomnika, 2019, 17(2), 1461-1467, doi: 10.12928/telkomnika.v17i3.10072.

[48] A. Gragera, and V. Suppakitpaisarn, In International Workshop on Algorithms and Computation, 2016, 339-350, doi: 10.1016/j.bspc.2016.06.012.

[49] X. Li, C. Wang, X. Zhang, and W. Sun, IEEE Access, 2020, 8, 66538-66552, doi: 10.1109/ACCESS.2020.2984024.

\section{Author Information}

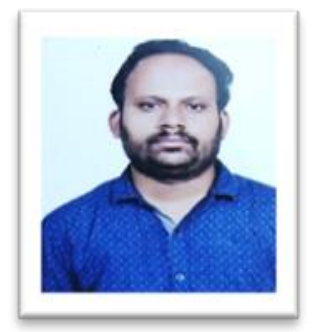

Rangayya has completed M.tech in Digital Electronics and communication Engineering from Visvesvaraya Technological University, Belagavi. Karnataka, INDIA, in the year 2009. Currently working as Assistant Professor in the department of Electronics and Communication Engineering, Sharnbasva University Kalaburagi. His research interest includes digital image processing, Signal Processing, Pattern Recognition, Computer Vision, Machine Learning etc.

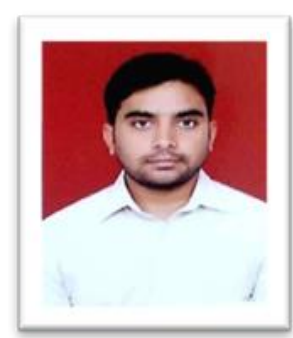

Dr. Virupakshappa received his Ph.D. from Visvesvaraya Technological University Belagavi in the year 2019 in the field of Biomedical Image Processing. Currently working as an Associate Professor in the department of Computer Science \& Engineering in the Sharnbasva University Kalaburagi. He has published several papers in the international journals and conferences. He has over ten years of teaching experience. His research interest include Digital Image Processing, Biomedical Image Processing, Pattern Matching, Machine Learning, Computer Vision etc.

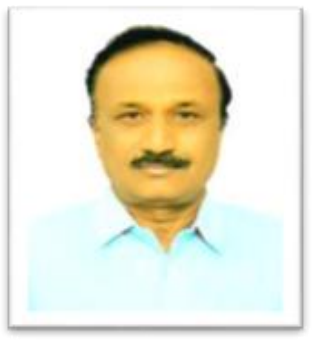

Dr. Nagabhushan Patil currently working as Head of the Department of Electrical \& Electronics Engineering in Poojya Doddappa College of Engineering Kalaburagi. He has received his Ph.D. in High Voltage Engineering in year 2017. He has thirty-five years of teaching experience. His area of research includes High voltage engineering, Analog Circuits, Digital Signal Processing, Digital Image Processing etc,.

Publisher's Note: Engineered Science Publisher remains neutral with regard to jurisdictional claims in published maps and institutional affiliations. 